\title{
Applying Improved Chaos System to Weak Signal Detection
}

\author{
Min Shi, Jin Chang Liu \\ Underwater Acoustic Antagonizing Laboratory, No. 91388 Troops of PLA, Zhanjiang, China \\ sarax@21cn.com, anliu@163.com
}

Keywords: Chaos, Improved chaos system, Weak signal detection

\begin{abstract}
Chaos system possesses the characteristics that it is immune to noise and signal whose frequency is quite different from that of inner signal in chaos system and it is sensitive to the signal whose frequency is same as that of inner signal in chaos system. Therefore the method for detection of weak signal hides in strong broad band background noise based on chaos theory has developed rapidly. But it finds in the actual simulating process that pure noise inputs into chaos system which is under critical state will probably induce to system state variation. And signal whose frequency is same as inner signal while phase is different from inner signal inputs into chaos system which is under critical state, the system state variation will probably not happen. The appearance of these cases reduced the detecting accuracy of weak signal. The setting method is proposed in the paper to the question. The amplitude of inner signal in chaos system is chosen to less than the threshold of chaos critical state, which can avoid system state variation induced by pure noise. Expanding the chaos system to four forms can detect input signal with different original phase. Simulation results verify the validity of the improved method.
\end{abstract}

\section{Introduction}

In the recent years, the method for weak signal detection based on chaos theory has developed rapidly and becomes a present hotspot in nonlinear research field [1-2]. It mains based on the characteristic that chaos system is immune to noise and signal whose frequency is very different from that of the inner signal in chaos system while it is sensitive to the signal whose frequency is same as that of inner signal in chaos system[3-8].

But in the actual simulation process, it finds when pure noise with some power inputs into the chaos system which is under critical state will probably induce the system into large scale period state [9-11]. And signal whose frequency is same as inner signal while phase is different from inner signal inputs into chaos system which is under critical state, the system state variation will probably not happen.

In order to resolve these questions, the improved chaos system detection method is used in the paper. The amplitude of inner signal in chaos system is chosen to less than the threshold of chaos critical state can avoid system state variation induced by pure noise. And expanding the chaos system to four forms can detect signal with different original phase from that of the inner signal in chaos system.

\section{Basic Principle of Weak Signal Detection Based on Chaos System}

Consider the Holmes duffing chaos system which is applied in weak signal detection for any frequency. The duffing equation is

$$
\ddot{x}=-k \omega \dot{x}+\omega^{2}\left(x-x^{3}+\gamma \cos (\omega t)\right)
$$

Where $\omega$ is angular frequency and $\gamma$ is amplitude of inner signals in chaos system, respectively. $k$ is damping ratio(usually $k=0.5$ ), $t$ is sampling time, $\dot{x}$ and $\ddot{x}$ are the first-order and second-order differential of $x$, respectively. $\left(x-x^{3}\right)$ is nonlinear restoring force.

With the variation of amplitude $\gamma$, the phase diagram of the system will go through different states. When $\gamma$ equals to chaos critical threshold $\gamma_{d}$, the system will enter into chaos critical state. At the 
moment, input signal whose frequency is same as that of inner signal, the system will transform from chaos state into large scale period state rapidly. While input pure noise, the system will keep the chaos state. Hereby weak signal can be detected by chaos system.

\section{Problem Existing in Signal Detection Based on Chaos System}

Suppose $\omega=1, k=0.5$, initialization value of the system is $[x, \dot{x}]=[1,1]$, according to Lyapunov exponent calculation or phase diagram of the system derived the chaos critical threshold $\gamma_{d}$ of equation (1) is 0.827 . Figure 1 gives the phase diagram of the system under $\gamma=0.827$ and $\gamma=0.828$, which verified the correction of the value of threshold $\gamma_{d}$.

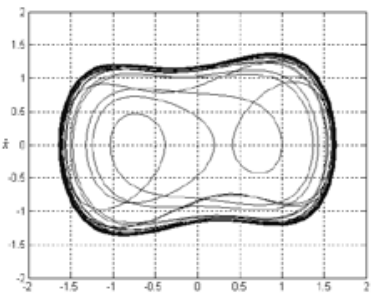

(a) $\gamma=0.827$

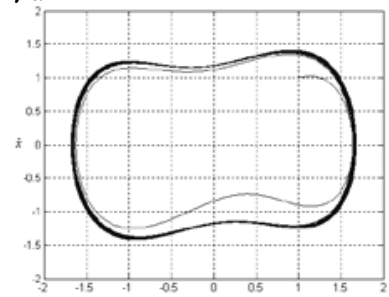

(b) $\gamma=0.828$

\section{Influence of Noise on Chaos System}

Fig. 1 The system phase contrail under different $\gamma$

Figure 2 gives the test result of chaos system under pure noise. (a) and (b) are the phase contrail diagram of chaos system when inputting gauss random white noise with power 0.1 into the chaos system under critical state. See from (b) that chaos system is not immune to noise absolutely. Sometimes the pure noise will induce the chaos system under critical state into large scale period state, which leads the method of signal detection by chaos system incorrectly.

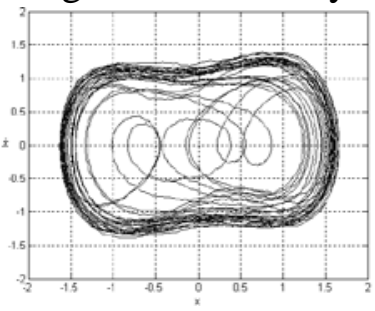

(a) noise power 0.1

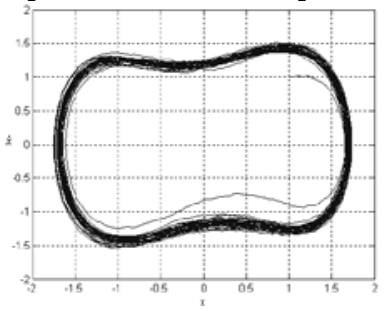

(b) noise power 0.1

Fig. 2 The system phase contrail after inputting signal with random noise

Influence of Orignial Phase of Signal on Chaos System

When inputting detection signal, the equation of chaos system is

$$
\ddot{x}=-k \omega \dot{x}+\omega^{2}\left(x-x^{3}+\gamma \cos (\omega t)+A \cos (\omega t+\psi)+n(t)\right)
$$

Where $A$ and $\psi$ is amplitude and original angle of the detection signal respectively, $n(\mathrm{t})$ is random noise. When $\gamma=\gamma_{d}$ in equation (2), input signal whose frequency is same as of that of inner signal in chaos system with $A=0.05$, the power of $n(\mathrm{t})$ is 0.5 . Figure 3 gives the system phase contrail diagram after inputting the detection signal, where (a) and (b) is corresponding to original phase $\psi=0$ and $\psi=$ $\pi$ respectively.

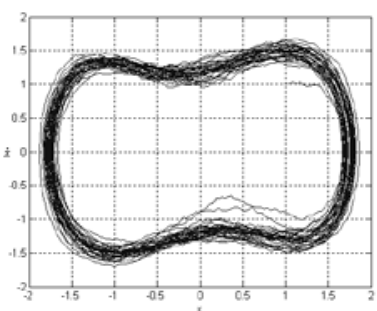

(a) $\psi=0$

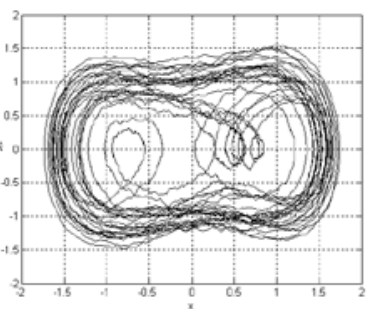

(b) $\psi=\pi$

Fig.3 The system phase contrail after inputting signal with random noise 
Show from figure 3 that original phase of signal can affect the detection result of chaos system. Sometimes the signal whose frequency is same as the inner signal but original phase is different from that of the inner signal will not induce the chaos system under critical state into large scale period state, which leads the method of signal detection by chaos system disabled.

\section{Basic Principle of Improved Chaos System}

Setting $\gamma$ value less than $\gamma_{d}$ in equation (2) can avoid the influence of noise on chaos detection system, for example, setting $\gamma=0.81$ in equation (2). After inputting signal, the inner signal of equation (2) becomes

$$
0.81 \cos (\omega t)+A \cos (\omega t+\psi)=\gamma_{D} \cos (\omega t+\theta)
$$

Where

$$
\begin{aligned}
& \gamma_{D}=\sqrt{0.81^{2}+1.62 A \cos \psi+A^{2}} \\
& \operatorname{tg} \theta=\frac{A \sin \psi}{0.81+A \cos \psi}
\end{aligned}
$$

Because for weak signal, $A<<0.81, \theta \approx 0$. The influence of $\theta$ on system can be ignored.

In theory, when $\gamma_{D}>\gamma_{d}$, system will go through from chaos state to large scale period state, which can detect the signal. Namely when satisfied

$$
\sqrt{0.81^{2}+1.62 A \cos \psi+A^{2}}>0.827
$$

The system will appear phase transformation.

If $A$ is known, the value range of $\psi$ can be calculated by equation (6). Suppose $A=0.035$, it can derive $-62.0172^{\circ}<\psi<62.0172^{\circ}$ by equation (6).Simulation result also shows when $\psi$ satisfied this condition, the state will transfer from chaos state to large scale state. In order to accurate detection, broaden the range of $\psi$ to $-60^{\circ} \leq \psi \leq 60^{\circ}$.From equation (6) can know when $A>0.035$, the range of $\psi$ will enlarged. So when amplitude of signal satisfied $A \geq 0.035$, the original angle of input signal satisfied $-60^{\circ} \leq \psi \leq 60^{\circ}$ will induce system phase transformation, so this type of signal can be detected. Other $\psi$ value will not induce the system transformation. If altering the “+” to "-” before inputting signal in equation (2), i.e duffing equation becomes

$$
\ddot{x}=-k \omega \dot{x}+\omega^{2}\left(x-x^{3}+\gamma \cos (\omega t)-A \cos (\omega t+\psi)-n(t)\right)
$$

The inner signal becomes

$0.81 \cos (\omega t)-A \cos (\omega t+\psi)=\gamma_{D 2} \cos (\omega t-\theta)$

Where $\gamma_{D 2}=\sqrt{0.81^{2}-1.62 A \cos \psi+A^{2}}, \theta \approx 0$ 。

In theory, when $\gamma_{D}>\gamma_{d}$, system will go through from chaos state to large scale period state, which can detect the signal. Namely the original angle of input signal satisfied $-180^{\circ} \leq \psi<-117.9828^{\circ}$ or $117.9828^{\circ}<\psi \leq 180^{\circ}$ can be detected by chaos system of equation (7). Broaden the range of $\psi$ to $-180^{\circ} \leq \psi \leq-120^{\circ}$ or $120^{\circ} \leq \psi \leq 180^{\circ}$.

The signal whose original angle satisfied $\psi \in[-\pi / 3, \pi / 3] \cup[-\pi,-2 \pi / 3] \cup[2 \pi / 3, \pi]$ can be detected by chaos system form of equation (2) and (7). For the signal whose original angle outside this range, phase compensation of inner signal can be introduced to resolve this question. Expand equation (2) to two forms

$$
\begin{aligned}
& \ddot{x}=-k \omega \dot{x}+\omega^{2}\left(x-x^{3}+\gamma \cos (\omega t+\pi / 2)+A \cos (\omega t+\psi)+n(t)\right) \\
& \ddot{x}=-k \omega \dot{x}+\omega^{2}\left(x-x^{3}+\gamma \cos (\omega t-\pi / 2)+A \cos (\omega t+\psi)+n(t)\right)
\end{aligned}
$$

The range of original angle can be detected by equation (8) is 
$\psi-\pi / 2 \in[-\pi / 3, \pi / 3]$, i.e $\psi \in[\pi / 6,5 \pi / 6]$, while the range of original angle can be detected by equation (9) is $\psi \in[-5 \pi / 6,-\pi / 6]$.Integrating the detection range of equation (2),(7),(8),(9) can cover the whole zone of $[-\pi, \pi]$.

The process of weak signal detection based on improved chaos system is listed as below

i ) Setting the amplitude of inner signal in chaos system is less than chaos critical state threshold, for example, $\gamma=0.81$.

ii ) Input the detection signal into equation (2),(7),(8),(9) respectively. If any phase contrail corresponding to the equations entered into large scale period state, it can conclude that detection signal has the same frequency as inner signal in chaos system.

\section{Simulation and Test}

Suppose the frequency of inner signal in equation (1) is $\omega=10$, when the initialization value of the system is $[x, \dot{x}]=[1,1]$, the chaos critical threshold is still 0.827 . Setting the amplitude of inner signal is $\gamma=0.81$. When inputting pure white noise, system phase transformation will not happen however the power of noise is. When inputting signal is $0.05 \cos \left(10 t+90^{\circ}\right)+n(\mathrm{t})(\mathrm{n}(\mathrm{t})$ is guass white noise with power 0.1 , the system phase contrail corresponding to equation (2),(7),(8),(9) is showed in figure4(a),(b),(c),(d) respectively.

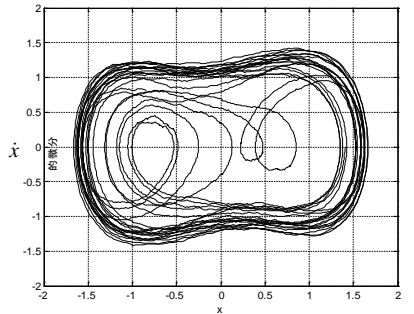

(a)

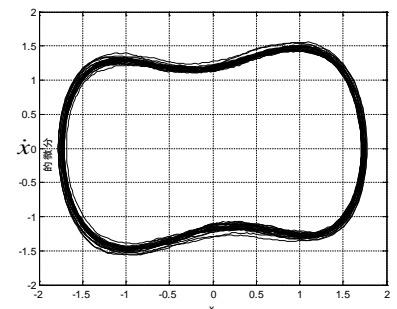

(c)

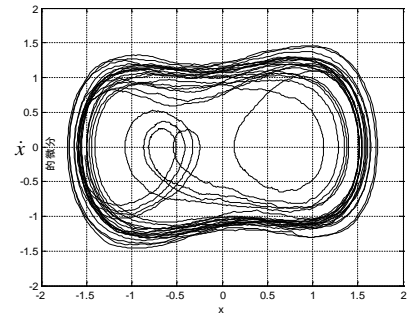

(b)

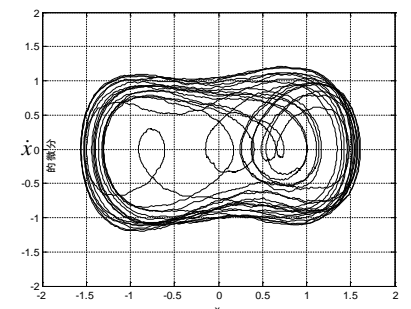

(d)

Fig. 4 system phase contrail diagram corresponding to equation (2),(7),(8),(9)

(a) system phase contrail diagram corresponding to equation (2), still in chaos state

(b) system phase contrail diagram corresponding to equation (7), still in chaos state

(c) system phase contrail diagram corresponding to equation (2), in large scale period state

(d) system phase contrail diagram corresponding to equation (2), still in chaos state

From figure 4 can see when inputting signal whose amplitude satisfied some condition (for example $A \geq 0.035$ ) while original phase is not 0 into the chaos system of equation (2), even though the frequency of signal is same as that of the inner signal, the system phase transformation will not happen, as figure 4(a) shows. After expanding equation (2) to four equations (2),(7),(8),(9), integrating the system phase contrail diagram corresponding to four equations can find the system state transformation, as figure 4 (c) shows. Figure 4 (b) and (d) denoted other two cases who cannot induce system phase transformation.

\section{Summary}

Weak signal detection based on improved chaos system method is proposed in the paper in order to avoid influence of noise and original phase of detection signal on test result. The amplitude of inner signal in chaos system is chosen to less than the threshold of chaos critical state can avoid system 
phase transformation induced by pure noise. Expanding the ordinal duffing equation to four different forms can detect signal whose amplitude satisfied some condition and any original phase. Simulation and test result showed that the improved method is effective. Next engineer apply of the method will be studied.

\section{References}

[1] T. L. Lou, Research on the detection of weak linear-spectrum signals based on chaotic theory, Ship Science and Technology, vol. 21, pp. 96-99, January 2009.

[2] S. Zhou and C.S.Lin, Application of chaos Theory for weak signal of ship detecting, Journal of Wuhan University of Technology (Transportation Science \& Engineering), vol. 33 , pp.161-164, January 2009.

[3] Y. F. Li and Y.F.Chen, "A method of weak vital periodic signals' frequency detection based on the chaotic oscillator,” Computer Engineering \& Science, vol. 33, pp. 183-189, May 2011.

[4] J. Chen, Application research and discussing of detective systems based on chaos theory, Journal of Gansu Gaoshi, vol. 18, pp. 21-25, February 2013.

[5] Y. X. Guo, Y.B. Liu and X.P.Wang Study on the fault diagnosis of rolling mills based on the chaos weak signal detection method, Journal of Engineering Design, vol. 18, pp. 218-221,232, March 2011.

[6] L.P. Zhu, L. Y.Zhang and W.F.Xie etal , Research of weak signal detection based on doffing chaotic oscillator, Radio Engineering, vol. 41, pp. 17-20, January 2012.

[7] Z. L. Ren, P. X. Liu and X. P. Shi, Improvement of weak sinusoidal signal detecting based on duffing oscillator, Computer Measurement \& Control, vol. 19, pp. 1301-1303, June 2011.

[8] L. Li, C. G. Liu and S. Shi etal, A method of weak signal detection based on chaotic oscillator and Lyapunov exponent, J Journal of Natural Science of Heilongjiang University, vol,29, pp.556-560, April 2012.

[9] Z. H. Lai, Y. G. Leng and J. Q. Sun, Weak characteristic signal detection based on scale transformation of doffing oscillator, Acta Phys.Sin., vol,61, 050503, May 2012.

[10]W.G.Chen, S.Zhang and L.Du etal, Photoacoustic Signal measuring based on cross-correlation analysis and chaotic detection, Electric Power Automation Equipment, vol.28, pp. 22-26, March 2012.

[11] L. Ran, M. Wang, Research on weak signal detection method under the strong noise background, Information Technology, , pp.41-45, February 2012. 